\title{
S76. Adoptive cell therapy of melanoma with autologous tumour infiltrating lymphocytes
}

\author{
I Svane ${ }^{*}$, R Andersen, M Donia \\ From 1st Immunotherapy of Cancer Conference (ITOC1) \\ Munich, Germany. 12-14 March 2014
}

\begin{abstract}
Background
Adoptive T-cell therapy (ACT) with tumor infiltrating lymphocytes (TILs) is a personalized treatment for cancer defined as the infusion of $\mathrm{T}$ cells isolated from the patient's own tumor tissue after ex vivo activation and several rounds of expansion. This treatment has achieved impressive clinical results in several single institution phase I/II clinical trials performed outside Europe, and holds the promise to enter the mainstream of standard melanoma care in the near future. However, although transient, the toxicities associated with high-dose IL-2 classically administered together with TILs are severe and recent data have questioned its use.
\end{abstract}

\section{Material and methods}

In an ongoing phase I/II study, we have enrolled patients with progressive metastatic melanoma. TILs infusion was preceded by standard lymphodepleting chemotherapy but followed by low-dose subcutaneous IL-2 for 14 days or by an intravenous intermediate dose IL-2 decrescendo regimen.

\section{Results}

The lower doses of IL-2 considerably decreased the toxicity of the treatment while PET-CT imaging showed a preserved objective response rate of $48 \%$ including longlasting complete responders. The absolute number of tumour specific T-cells infused was significantly associated to clinical response, with induction of peripheral tumour reactive $\mathrm{T}$ cells. To characterize the fate of TILs after infusion, we performed a longitudinal analysis of bulk tumor-reactive $\mathrm{T}$ cells from in vitro cultured TILs and found durable persistent $\mathrm{T}$-cells after up to 1.5 years after infusion. Extensive analysis of disease relapse in two patients revealed multiple potential mechanisms of

Copenhagen University Hospital, Department of Oncology Center for Cancer Immune Therapy, Herlev, Denmark 